\title{
Dieta estival del piuquén (Chloephaga melanoptera Eyton, 1838) en praderas hidromórficas de la Cordillera Andina de la Región de Coquimbo, Chile
}

\author{
Summer diet of the piuquén (Chloephaga melanoptera Eyton, 1838) in \\ hydromorphic grasslands of the Andean Mountain of the Coquimbo Region, Chile \\ Carla Orellana $^{1 *}$, Giorgio Castellaro ${ }^{1}$, Juan Escanilla ${ }^{1}$
}

\begin{abstract}
RESUMEN
El objetivo del trabajo fue estudiar la dieta del piuquén en praderas hidromórficas de altura, en la Región de Coquimbo, durante dos temporadas, a través de su composición botánica, estableciendo relaciones entre la alimentación y ciertos atributos del pastizal (producción de materia seca, diversidad, contribución de Eleocharis pseudoalbibracteata y Carex spp.). La dieta fue analizada mediante microhistología de heces y la caracterización del pastizal a través del método de Point Quadrat para la composición botánica y con el uso de exclusiones para estimar producción de materia seca. Nuestros resultados muestran que esta ave selecciona esencialmente especies gramíneas y graminoides, en especial E. pseudoalbibracteata, presentando valores de selectividad significativos y contribuciones en la dieta de hasta $48 \%$, lo que se relacionó directamente con la diversidad del pastizal $(r=0,80$; $\mathrm{P}=0,0078)$ y con la presencia de la misma especie en el recurso forrajero $(\mathrm{r}=0,75 ; \mathrm{P}=0,0123)$. No se encontraron diferencias entre temporadas para los grandes grupos de especies evaluadas en la dieta; sin embargo, los cambios significativos en la contribución de Carex spp. y E. pseudoalbibracteata indicarían que la modificación de la conducta de forrajeo sería en función de la ingesta de proteína de alta disponibilidad presente en algunas especies vegetales dentro del grupo de graminoides.
\end{abstract}

Palabras clave: Carex spp., Eleocharis pseudoalbibracteata, gansos silvestres, herbivoría, veranadas de montaña.

\section{ABSTRACT}

The objective of this work was to study the diet of Piuquén in wet highland grasslands, in the Coquimbo Region, during two seasons through its botanical composition, establishing relationships between diet and certain attributes of the pasture (dry matter production, diversity, contribution of Eleocharis pseudoalbibracteata and Carex spp). The diet was analyzed by fecal microhistology, and the characterization of the pasture through the Point Quadrat method for the botanical composition and the use of exclusions to estimate dry matter production. Our results evince that this bird essentially selects grasses and graminoids, especially E. pseudoalbibracteata presenting significant selectivity values and contributions in the diet of up to $48 \%$, which was directly related to pasture diversity $(r=0.80 ; P=0.0078)$ and with the presence of the same species in the forage resource $(r=0.75, P=0.0123)$. No differences were found between seasons for the large groups of species evaluated in the diet, however the significant changes in the contribution of Carex spp. and E. pseudoalbibracteata, would indicate that the modification of the foraging behavior would be in function of the intake of high availability protein present in some plant species within the group of graminoides.

Keywords: Carex spp., E. pseudoalbibracteata, herbivory, mountain summer range, wild geese.

\section{Introducción y revisión bibliográfica}

El piuquén (Chloephaga melanoptera, Eyton 1838; familia Anatidae) es una especie de ganso silvestre que habita humedales altoandinos de la puna en los Andes de Perú, Bolivia, Chile y Argentina (Anderson et al., 2012), donde convive con otros herbívoros silvestres (guanacos) y domésticos (equinos, caprinos), usando el mismo recurso forrajero durante el periodo estival. Los humedales altoandinos son sistemas que mantienen una diversidad biológica única y se caracterizan por un alto grado de endemismo de plantas y animales (García et al., 2015). Poseen un estrato herbáceo denso, con una cantidad importante de especies palatables y, por tanto, relevantes para la

\footnotetext{
1 Departamento de Producción Animal, Facultad de Ciencias Agronómicas de la Universidad de Chile. Santiago de Chile, Chile.

* Autor por correspondencia: carlaorellana@ug.uchile.cl.
} 
nutrición de los individuos que las utilizan (Cochi et al., 2014), transformándose en ecosistemas claves para la conservación de las aves asociadas a ambientes húmedos (Tellería et al., 2006). Tanto en patos como en gansos silvestres, el tamaño del tracto digestivo y la reducida capacidad de digerir los alimentos fibrosos podrían condicionar su estatus nutritivo respecto de otros herbívoros como caprinos o guanacos, que tienen mayor capacidad de consumo de materia seca y un sistema digestivo adaptado para utilizar forrajes de regular calidad (Durant et al., 2004; Ruifrok et al., 2015). Bajo esta perspectiva, en gansos, la selectividad de aquellos ítems alimentarios altamente proteicos se torna de vital importancia, y es incluso más relevante que la cantidad total de alimento consumido (Doiron et al., 2014). Dicha capacidad de selección diferiría de la que presentan otros herbívoros de distinto tamaño corporal, debido a sus disímiles niveles de requerimientos metabólicos y habilidades para cosechar y digerir el alimento (Wang et al., 2013). Además, es importante considerar el efecto que tienen sobre el patrón de alimentación de los individuos, las variaciones que presenta el recurso forrajero en función de las condiciones ambientales imperantes, las cuales muchas veces son más relevantes que los propios requerimientos nutricionales (Barboza et al., 2009), condicionando el uso del recurso trófico disponible. Respecto de los hábitos dietarios del piuquén en ambientes altoandinos, Summers y Castro (1988) mencionan algunas especies vegetales que serían relevantes para esta ave en ecosistemas peruanos; sin embargo, no se presentan análisis de selectividad o la asociación que podría existir entre la dieta y el recurso. Un primer paso para comprender las interacciones que pueden manifestarse entre las especies de herbívoros que habitan estos humedales, es conocer la dieta y su relación con el recurso forrajero disponible. Por ello, el objetivo de este trabajo fue caracterizar la dieta del piuquén en términos de su composición botánica y diversidad, además de conocer el efecto que podrían tener ciertos atributos del pastizal, como la producción de materia seca y la contribución de las especies vegetales más importantes, sobre el patrón de alimentación de esta ave.

\section{Materiales y métodos}

El estudio se realizó durante dos temporadas (2011/2012), en seis sitios de pastizales hidromórficos de altura (comúnmente denominados "humedales" o "vegas"), localizados en áreas depresionales de la cordillera de Cuncumén (IV Región de Coquimbo, comuna de Salamanca, Chile $31^{\circ} 52^{\prime}-32^{\circ} 02^{\prime}$ lat. S; 70¹9'-70²6' long. O; 3025-3729 msnm), El sector posee un clima estepario muy frío, con precipitaciones que ocurren mayormente entre abril y septiembre (91\%), con un promedio de $329,3 \pm 153,5 \mathrm{~mm}$, y que caen en un $91 \%$ en forma de nieve. La temperatura media anual es del orden de los $6,8^{\circ} \mathrm{C}$. El mes más cálido es enero $\left(11,2^{\circ} \mathrm{C}\right)$ y el más frío julio $\left(2,8^{\circ} \mathrm{C}\right)$. Según la clasificación climática de Köeppen, el clima del sector es del tipo BSsk'. Los suelos donde crecen los pastizales hidromórficos ("vegas") ocupan una posición de fondo de quebrada, con pendiente plana-suave $(<4,5 \%)$ a suave-inclinada $(4,5-10,5 \%)$, de textura franco arenosa fina a arenosa fina y con profundidad efectiva de $50 \mathrm{~cm}$ o más. Presentan un grado de hidromorfismo permanente, con exceso de agua en el perfil de suelo en gran parte del año (Gastó et al., 1993). Sobre estos suelos se desarrolla una vegetación hidrofítica de alta cobertura, donde destacan gramíneas como Deschampsia cespitosa y Deyeuxia vetulina. En otros sitios y a mayor altitud, dominan juncáceas en cojín como Oxychloe andina y Patosia clandestina, además de una serie de especies secundarias importantes como ciperáceas de los géneros Carex y Eleocharis, junto con diversas especies del género Juncus (Squeo et al., 2006).

La producción de materia seca fue estimada con el uso de 22 exclusiones de $7,29 \mathrm{~m}^{2}$ de área útil, dispuestas a inicios de la estación de crecimiento (diciembre) en los seis sitios de vega (Vega La Redonda, Estero El Bonito, Vega Las Mesas,; Vega Alitre Alto, Vega Alitre Bajo, Vega La Rajada Baja). Todo el material vegetal acumulado al final de la temporada de crecimiento (fines de marzo) fue pesado en fresco y posteriormente deshidratado en una estufa con aire forzado a $60^{\circ} \mathrm{C}$, hasta peso constante (AOAC, 2000).

La composición botánica en cada sitio de vega fue determinada a través del método de Point Quadrat (Daget y Poissonet, 1971), utilizando 2 transectos de $20 \mathrm{~m}$ asociados a cada exclusión. En cada transecto se evaluaron 100 puntos, diferenciando el número de toques efectuados sobre las diferentes especies vegetales, tanto en estructuras forrajeables como en no forrajeables, así como mantillo, rocas y piedras y suelo desnudo (Passera et al., 1983). La información 
anterior fue utilizada para calcular la diversidad y la contribución de las especies vegetales Carex spp. (C. maritima, C. gayana y C. vallis-pulchrae) y Eleocharis pseudoalbibracteata) en cada uno de los seis sitios de vega evaluados. Paralelamente, se recolectaron muestras de heces frescas de piuquén en cada sector de vega, constituidas por un conjunto de 10 a 15 submuestras, las cuales fueron recogidas haciendo un recorrido sistemático en toda el área de influencia de cada vega.

La composición botánica de la dieta se determinó mediante análisis microhistológico de heces (Garnick et al., 2018). Para la obtención y reconocimiento de la epidermis de las especies vegetales se utilizaron las técnicas propuestas por Castellaro et al. (2007). Las especies fueron observadas bajo un microscopio óptico (Olympus, modelo CX21), con cámara digital incorporada, utilizando aumentos de 100X y 400X, y caracterizadas siguiendo los criterios y la nomenclatura recomendada por Ortega et al. (1993).

El resultado de la lectura microscópica se expresó como frecuencia relativa y posteriormente transformada en densidad, utilizando las tablas propuestas por Fracker y Brischle (1944) (citados por Ortega et al., 1993). Las especies identificadas fueron agrupadas en cuatro grandes grupos: gramíneas (poáceas), graminoides (ciperáceas y juncáceas), hierbas dicotiledóneas y especies arbustivas. La diversidad del pastizal y de la dieta se determinó mediante el cálculo del índice de Shannon - Wiener $\left(\mathrm{H}=-\sum_{\mathrm{i}=1}^{\mathrm{n}} \mathrm{P}_{\mathrm{i}} \cdot \log _{2}\left(\mathrm{P}_{\mathrm{i}}\right)\right)$, el cual se expresó como diversidad relativa o equiparidad $\left(\mathrm{J}=\frac{\mathrm{H}}{\mathrm{H}_{\max }}\right)$ (Smith y Smith, 2012).

En las ecuaciones anteriores $P_{i}$ es la proporción de la especie i en el pastizal/dieta y $n$ es el número de especies en la misma. $H_{\max }$, representa el valor que tendría $H$ si todas las especies encontradas en el pastizal/dieta tuviesen la misma frecuencia $\left(H_{\text {max }}=\log _{2}(n)\right)$.

El grado de selección de las principales especies vegetales que compusieron la dieta fue establecido utilizando el índice de selección de Savage $\left(\mathrm{W}_{\mathrm{i}}=\frac{\mathrm{U}_{\mathrm{i}}}{\mathrm{P}_{\mathrm{i}}}\right)\left(\right.$ Savage, 1931), donde $U_{i}$ corresponde a la proporción de una especie vegetal i en la dieta y $P_{i}$ es la proporción de la misma especie en el pastizal. Este índice toma valores que van de 0 (máximo rechazo) a infinito (máxima selección), donde 1 indica que existe indiferencia por el consumo de un ítem en particular, siendo utilizado al azar. El índice de selección de Savage permite calcular un estadístico $\mathrm{E}$ con una distribución $\lambda^{2}$ con un grado de libertad $(\alpha=0,05)\left(\mathrm{E}_{\mathrm{i}}=\frac{\left(\mathrm{W}_{\mathrm{i}}-1\right)^{2}}{\mathrm{ee}\left(\mathrm{W}_{\mathrm{i}}\right)^{2}}\right)$, indicando si el valor de selectividad obtenido es significativo (Manly et al., 1993). En la ecuación anterior, $e e\left(W_{i}\right)$ es el error estándar del índice de Savage $\left(\operatorname{ee}\left(\mathrm{W}_{\mathrm{i}}\right)=\sqrt{\frac{\left(1-\mathrm{P}_{\mathrm{i}}\right)}{\left(\mathrm{u}-\mathrm{P}_{\mathrm{i}}\right)}}\right)$, donde $u$ corresponde al número total de fragmentos identificados en las heces al realizar el análisis microhistológico.

La contribución de las especies gramíneas, graminoides, hierbas dicotiledóneas y arbustivas en la dieta, así como el índice de diversidad de esta $(\mathrm{J})$, fueron analizados mediante un modelo lineal generalizado, considerando la temporada de evaluación como la principal fuente de variación. Luego se realizó un test LSD con un $95 \%$ de confianza para la separación de medias. De esta misma forma se evaluó la contribución de las especies Carex spp. y E. pseudoalbibracteata, en función de la alta contribución de ambas especies observadas en la dieta.

Para establecer las asociaciones entre los resultados del análisis microhistológico de heces y la caracterización del pastizal se calculó una matriz de correlación de Spearman con 95\% de significancia entre la diversidad, contribución de las especies Carex spp. y E. pseudoalbibracteata de la dieta y la diversidad, producción de materia seca y contribución de Carex spp. y E. pseudoalbibracteata del pastizal, por ser estas dos especies las más relevantes en la composición de los pastizales evaluados.

Para todos los análisis estadísticos mencionados se utilizó el programa STATGRAPHICS Centurion XV.II.

\section{Resultados y discusión}

En ambos periodos, la composición botánica del pastizal estuvo dominada por especies graminoides (>55\%), donde Carex spp., E. pseudoalbibracteata y $P$. clandestina fueron las que presentaron mayor contribución, observando una media a alta diversidad, con producciones de materia seca entre 1056 y $1226 \mathrm{~kg} \mathrm{ha}^{-1}$ (Tabla 1). Dentro del grupo de las gramíneas, segundo más importante en la 
composición botánica del pastizal, destacaron las especies Deyeuxia chrysostachya y Festuca werdermannii. El grupo de dicotiledóneas herbáceas tuvo una participación cercana al 18\%, siendo las especies Cardamine volckmanni, Plantago barbata y Lobelia oligophylla las de mayor contribución (Tabla 1). En general, dicha composición botánica es similar a la descrita por Squeo et al. (2006) en relación con los géneros más importantes que componen este tipo de pastizales, los que están dominados fundamentalmente por especies graminoides, y los niveles productivos

Tabla 1. Composición botánica del pastizal (promedio porcentual \pm error estándar) y su respectivo índice de diversidad y producción de materia seca. Cuncumén, IV Región de Coquimbo, Comuna de Salamanca, Chile (Temporadas 2011-2012).

\begin{tabular}{|c|c|c|}
\hline \multirow[b]{2}{*}{ Especie vegetal } & \multicolumn{2}{|c|}{ Temporadas } \\
\hline & 2011 & 2012 \\
\hline Deyeuxia chrysostachya E. Desv. & $9,98 \pm 5,22$ & $3,13 \pm 1,34$ \\
\hline Festuca werdermannii St.-Yves. & $7,41 \pm 4,46$ & $0,10 \pm 0,08$ \\
\hline Deyeuxia erythrostachya E.Desv. & $4,49 \pm 2,15$ & - \\
\hline Hordeum pubiflorum Hook F. & $3,71 \pm 2,37$ & $0,46 \pm 0,32$ \\
\hline Nicoraepoa subenervis var. Spegazzini Soreng \& L.J & $0,46 \pm 0,46$ & - \\
\hline Trisetum sp. & $0,04 \pm 0,04$ & - \\
\hline Deschampsia caespitosa (L.) P. Beauv. & $0,16 \pm 0,11$ & $0,81 \pm 0,81$ \\
\hline Deschampsia pulchra Nees \& Meyen. & - & $0,63 \pm 0,54$ \\
\hline Festuca panda Swallen. & - & $2,24 \pm 2,24$ \\
\hline Phleum alpinum $\mathrm{L}$. & - & $0,31 \pm 0,17$ \\
\hline Total Gramíneas & $26,26 \pm 8,11$ & $7,68 \pm 2,54$ \\
\hline Carex spp. ${ }^{1}$ & $31,75 \pm 6,53$ & $27,01 \pm 3,64$ \\
\hline Eleocharis pseudoalbibracteata Nees \& Meyen ex Kunth. & $11,91 \pm 3,44$ & $33,51 \pm 3,79$ \\
\hline Patosia clandestina (Phil.) Buchenau. & $11,22 \pm 4,11$ & $7,79 \pm 2,97$ \\
\hline Juncus arcticus var. Andicola Willd. & $0,36 \pm 0,36$ & $2,63 \pm 1,52$ \\
\hline Zameioscirpus gaimarioides (É.Desv.) Dhooge \& Goetgh & $0,22 \pm 0,15$ & $3,96 \pm 1,40$ \\
\hline Phylloscirpus acaulis Phill. & - & $0,14 \pm 0,14$ \\
\hline Total Graminoides & $55,44 \pm 7,44$ & $75,03 \pm 5,17$ \\
\hline Cardamine volckmannii Phill. & $4,97 \pm 2,77$ & - \\
\hline Plantago barbata $\mathrm{G}$. Forst & $4,53 \pm 3,45$ & $0,53 \pm 0,32$ \\
\hline Lobelia oligophylla Wedd. & $3,61 \pm 1,49$ & $4,93 \pm 1,54$ \\
\hline Azorella trifoliolata Clos. & $1,79 \pm 0,91$ & $2,22 \pm 1,43$ \\
\hline Trifolium repens $\mathrm{L}$. & $0,66 \pm 0,42$ & $0,41 \pm 0,39$ \\
\hline Stellaria debilis d'Urv. & $0,61 \pm 0,39$ & $0,20 \pm 0,20$ \\
\hline Werneria pygmaea Gillies ex Hook. \& Arn. & $0,55 \pm 0,33$ & $2,82 \pm 1,25$ \\
\hline Mimulus depressus Phill. & $0,44 \pm 0,35$ & $0,04 \pm 0,04$ \\
\hline Gentiana prostrata Haenke. & $0,42 \pm 0,23$ & - \\
\hline Cerastium humifusum Cambess. ex A. St.-Hil. & $0,29 \pm 0,29$ & $0,13 \pm 0,07$ \\
\hline Erigeron andicola DC. & $0,21 \pm 0,14$ & $0,95 \pm 0,81$ \\
\hline Ranunculus Cimbalaria Pursh. & $0,15 \pm 0,12$ & $0,35 \pm 0,33$ \\
\hline Taraxacum officinale G.H. Weber ex Wigg & $0,06 \pm 0,06$ & $0,09 \pm 0,06$ \\
\hline Calceolaria filicaulis ssp. Luxurians Witasek & $0,04 \pm 0,04$ & - \\
\hline Plantago uniglumis Wallr. ex Walp. & - & $2,72 \pm 2,46$ \\
\hline Calandrinia affinis Gillies ex Arn. & - & $1,26 \pm 1,10$ \\
\hline Olsynium philippi (Klatt) Goldblatt & - & $0,59 \pm 0,59$ \\
\hline Cardamine cfr. Vulgaris Phill. & - & $0,03 \pm 0,03$ \\
\hline Gayophytum micranthum Hook. et Arn. & - & $0,02 \pm 0,02$ \\
\hline Total dicotiledóneas herbáceas & $18,32 \pm 5,40$ & $17,29 \pm 3,80$ \\
\hline Índice de diversidad del pastizal & $0,675 \pm 0,033$ & $0,815 \pm 0,033$ \\
\hline Producción de materia seca $\left(\mathrm{kg} \mathrm{ha}^{-1}\right)$ & $1056,2 \pm 306,9$ & $1226,2 \pm 306,9$ \\
\hline
\end{tabular}


son concordantes con el aporte hídrico, lluvia y deshielos (Le Huerou, 1984).

Todos los fragmentos epidemiales identificados en la dieta correspondieron a tejidos vegetales, sin observarse diferencias entre temporadas en cuanto a la contribución de los grandes grupos de especies ( $p \geq 0,05)$, donde las graminoides fueron las más importantes y constituyeron más del $50 \%$ de la dieta en ambos periodos. Se destacaron las especies Carex spp. y E. pseudoalbibracteata , y esta última mostró una alta contribución en la dieta durante la temporada 2012 ( $\mathrm{p} \leq 0,05)$. En el caso de las especies gramíneas, dicotiledóneas herbáceas y plantas arbustivas, no se destacó ninguna especie en particular (Tabla 2). Summers y Castro (1988), quienes trabajaron con la dieta de esta misma ave pero en la zona altiplánica del Perú (4078 msnm), y Summers y Grieve (1982), que estudiaron los hábitos dietarios de Chloëphaga picta leucoptera (Gmelin) en las Islas Malvinas, coinciden en que la dieta de estas aves es esencialmente herbívora (hojas y tallos). Sin embargo, en ambos trabajos, las especies vegetales mayormente consumidas fueron Poa annua y $P$. pratensis, pertenecientes al grupo de las gramíneas, en tanto que en este estudio las especies más importantes fueron Carex spp. y E. pseudoalbibracteata, ambas graminoides pertenecientes a la familia Cyperaceae. Al respecto, se ha reportado la relevancia de algunas especies graminoides como Carex subspathacea, Puccinellia phryganodes y Scirspus sp. en la dieta del ganso Anser caerulescens caerulescens (Alisauskas et al., 1988), así como la alta contribución de $C$. aquatilis y Scirpus sp. en la dieta de A. caerulescens atlanticus y A. anser (Gauthier, 1993). Al margen de la especie de gramínea o graminoide que domine la dieta del piuquén, la suma de estos grupos vegetales contribuyó con un porcentaje cercano al 89\%, mayor a lo indicado por Karmiris et al. (2011) para el ganso A. albifrons, quienes reportan valores del orden de $61 \%$.

Aparentemente los individuos eligen de forma constante aquellos grupos de plantas que maximicen la ingesta de energía y proteína, con contenidos bajos de fibra, dado que su aparato digestivo se caracteriza por poseer una reducida capacidad de uso de los componentes fibrosos vegetales (Sedinger, 1997). Análisis químicos realizados en muestras de Carex spp. indican concentraciones de proteína bruta de $10,5 \%$, en tanto que para E. tucumanensis, especie similar y perteneciente a la misma familia que E. pseudoalbibracteata, dicha concentración es del orden de 12,2\% (Castellaro et al., 2012). Ambos contenidos de proteína bruta estarían por sobre el promedio estimado para toda la vega $(\sim 8 \%)$, lo que podría explicar la alta contribución de ambas especies vegetales en la dieta. Al respecto, Durant et al. (2004) indican que, en general, las aves herbívoras maximizan su tasa de ingesta de nitrógeno digestible en lugar de la ingesta de materia seca, por lo que la concentración de nitrógeno de las plantas es más importante que la cantidad de fitomasa total (Doiron et al., 2014).

El índice de diversidad dietaria $\left(\mathrm{J}_{\mathrm{d}}\right)$ fue mayor durante el año 2011, con un valor de 0,69 en contraste con 0,49 obtenido en la temporada 2012 $(\mathrm{p} \leq 0,05)$ (Tabla 2), observándose una correlación negativa tanto con la diversidad de la vegetación $\left(\mathrm{J}_{\mathrm{veg}}\right)(\mathrm{p} \leq 0,05)$ como con la contribución de E. pseudoalbibracteata del pastizal $(\mathrm{p} \leq 0,05)$. La participación de Carex spp. en la dieta también presentó una correlación negativa con $\mathrm{J}_{\mathrm{veg}} \mathrm{y}$ con la contribución de E. pseudoalbibracteata del pastizal $(\mathrm{p} \leq 0,05)$. Contrario a lo observado anteriormente, la participación de E. pseudoalbibracteata en la dieta mostró correlaciones positivas con $\mathrm{J}_{\text {veg }} \mathrm{y}$ con la contribución de E. pseudoalbibracteata del pastizal $(\mathrm{p} \leq 0,05)$ (Tabla 3). Ni la producción de materia seca ni la contribución de Carex spp. en el pastizal presentaron correlaciones significativas con las demás variables evaluadas (Tabla 3).

Del total de especies que mostraron un alto consumo ( $>15 \%$ de aporte en la dieta), solo E. pseudoalbibracteata fue seleccionada de forma significativa, especialmente durante la segunda temporada, y presentó una contribución importante en la dieta (Tabla 4). Wang et al. (2013) indican que esta especie vegetal muestra una mayor facilidad en la extracción de nitrógeno $(\mathrm{N})$ desde los tejidos vegetales, en relación con otras especies como Carex spp., puesto que dichos tejidos son menos duros y fibrosos. $\mathrm{La}$ diferencia entre el perfil aminoacídico del recurso alimenticio y sus propios requerimientos hace que los gansos sean altamente selectivos (Durant et al., 2004), priorizando el consumo de plantas o partes de plantas que tengan muchas proteínas y bajo contenido de fibra (López-Calleja y Bozinovic, 2000).

Estos antecedentes podrían explicar tanto el índice de selección como los valores en las correlaciones encontradas entre la presencia de E. pseudoalbibracteata en el pastizal y los distintos atributos dietarios evaluados (Tabla 3), así como 
Tabla 2. Composición botánica de la dieta del piuquén (Chloephaga melanoptera Eyton, 1838)

(promedio porcentual \pm error estándar) y su respectivo índice de diversidad. Cuncumén,

IV Región de Coquimbo, comuna de Salamanca, Chile (Temporadas 2011-2012).

\begin{tabular}{|c|c|c|}
\hline \multirow[b]{2}{*}{ Especie vegetal } & \multicolumn{2}{|c|}{ Temporada } \\
\hline & 2011 & 2012 \\
\hline Festuca sp. & $5,03 \pm 4,18$ & $0,14 \pm 0,07$ \\
\hline Trisetum oreophilum var. Johnstonii Louis-Marie. & $3,45 \pm 2,00$ & $0,18 \pm 0,14$ \\
\hline Nicoraepoa aff subenervis var. Spegazzini Soreng \& L.J. & $2,18 \pm 1,98$ & $8,05 \pm 8,03$ \\
\hline Panicum sp. & $1,67 \pm 1,25$ & - \\
\hline Distichlis sp. & $1,64 \pm 1,64$ & - \\
\hline Deyeuxia chrysostachya E. Desv. & $1,63 \pm 0,72$ & $0,17 \pm 0,11$ \\
\hline Hordeum pubiflorum Hook F. & $1,24 \pm 0,63$ & $5,00 \pm 2,13$ \\
\hline Bromus tunicatus Phil. & $1,06 \pm 0,55$ & $0,04 \pm 0,04$ \\
\hline Festuca werdermannii St.-Yves & $0,81 \pm 0,32$ & - \\
\hline Deyeuxia aff. Erythrostachya E. Desv. & $0,72 \pm 0,35$ & $0,21 \pm 0,08$ \\
\hline Festuca panda Swallen. & $0,65 \pm 0,61$ & $0,03 \pm 0,03$ \\
\hline Deschampsia caespitosa (L.) P. Beauv. & $0,38 \pm 0,22$ & $0,02 \pm 0,02$ \\
\hline Trisetum preslei (Kunth) E. Devs. & $0,20 \pm 0,20$ & $0,05 \pm 0,05$ \\
\hline Stipa chrysophylla E. Desv. & $0,15 \pm 0,10$ & $0,04 \pm 0,04$ \\
\hline Phleum alpinum $\mathrm{L}$. & - & $0,83 \pm 0,75$ \\
\hline Deyeuxia velutina Nees \& Meyen. & - & $0,52 \pm 0,47$ \\
\hline Bromus sp. & - & $0,44 \pm 0,27$ \\
\hline Deyeuxia sp. & - & $0,04 \pm 0,04$ \\
\hline Otras Gramíneas No Identificadas & $6,02 \pm 1,56$ & $3,93 \pm 1,40$ \\
\hline Total Gramíneas & $26,84 \pm 6,69 a$ & $19,68 \pm 9,82 \mathrm{a}$ \\
\hline Carex spp. ${ }^{1}$ & $30,24 \pm 8,40 \mathrm{a}$ & $18,16 \pm 5,77 \mathrm{~b}$ \\
\hline Eleocharis pseudoalbibracteata Nees \& Meyen ex Kunth. & $1,62 \pm 1,09 \mathrm{~b}$ & $47,62 \pm 13,25 \mathrm{a}$ \\
\hline Patosia clandestina (Phil.) Buchenau & $13,71 \pm 7,67$ & $6,17 \pm 3,33$ \\
\hline Oxychloe andina Phill. & $3,50 \pm 3,00$ & $0,04 \pm 0,04$ \\
\hline Zameioscirpus gaimardioides (É.Desv.) Dhooge \& Goetgh. & $3,36 \pm 1,43$ & $0,09 \pm 0,06$ \\
\hline Juncus arcticus Willd. & $2,12 \pm 1,09$ & $0,04 \pm 0,04$ \\
\hline Juncus sp. & - & $1,66 \pm 1,19$ \\
\hline Phylloscirpus acaulis Phill. & - & $0,13 \pm 0,11$ \\
\hline Otros Graminoides No Identificados & $0,67 \pm 0,35$ & $1,30 \pm 0,91$ \\
\hline Total Graminoides & $55,23 \pm 12,03 a$ & $75,21 \pm 9,74 a$ \\
\hline Montiopsis potentilloides (Barneoud) Ford. & $1,31 \pm 1,19$ & $0,06 \pm 0,04$ \\
\hline Gayophytum micranthum Hook. Et Arn. & $0,90 \pm 0,82$ & - \\
\hline Jaborosa laciniata (Miers) Hunz. & $0,87 \pm 0,53$ & $0,09 \pm 0,09$ \\
\hline Werneria pygmaea Gillies ex Hook. \& Arn. & $0,83 \pm 0,83$ & - \\
\hline Trifolium repens $\mathrm{L}$. & $0,64 \pm 0,43$ & $0,02 \pm 0,02$ \\
\hline Cardamine cfr. Vulgaris Phill. & $0,61 \pm 0,61$ & $0,18 \pm 0,12$ \\
\hline Mimulus depressus Phill. & $0,61 \pm 0,61$ & - \\
\hline Cerastium arvense $\mathrm{L}$. & $0,18 \pm 0,11$ & $0,19 \pm 0,08$ \\
\hline Calceolaria filicaulis ssp. Luxurians Witasek. & $0,05 \pm 0,05$ & - \\
\hline Chaetanthera euphrasioides DC. & $0,05 \pm 0,12$ & - \\
\hline Gentiana prostrata Haenke. & $0,10 \pm 0,10$ & $0,03 \pm 0,03$ \\
\hline Lobelia oligophylla Wedd. & $0,10 \pm 0,10$ & $0,23 \pm 0,13$ \\
\hline Chaetanthera pulvinata Phill. & - & $0,03 \pm 0,03$ \\
\hline Perezia carthamoides (D. Don) Hook. \& Arn. & - & $0,04 \pm 0,04$ \\
\hline Phacelia secunda J.F. Gmelin. & - & $0,04 \pm 0,04$ \\
\hline Ranunculus Cimbalaria Pursh. & - & $0,56 \pm 0,43$ \\
\hline Dicotiledoneas no Identificadas. & $3,85 \pm 1,73$ & $3,58 \pm 0,73$ \\
\hline Total Dicotiledóneas & $10,09 \pm 4,48 \mathrm{a}$ & $5,05 \pm 1,09 a$ \\
\hline Berberis empetrifolia Lam. & $3,06 \pm 1,82$ & - \\
\hline Adesmia sp. & $2,69 \pm 2,29$ & $0,06 \pm 0,04$ \\
\hline Chuquiraga oppositifolia D. Don. & $0,20 \pm 0,20$ & - \\
\hline Nassauvia cumingii Hook. \& Arn. & $0,20 \pm 0,20$ & - \\
\hline Total Arbustivas & $6,16 \pm 4,27 a$ & $0,06 \pm 0,04 a$ \\
\hline Índice de diversidad de la dieta & $0,698 \pm 0,131 \mathrm{a}$ & $0,493 \pm 0,159 b$ \\
\hline
\end{tabular}

1 Carex spp. se refiere a la sumatoria de las especies Carex gayana Desv., Carex maritima Gunn. y Carex vallis-pulchrae Phil.

Letras distintas entre columnas indican diferencia estadística significativa entre las temporadas evaluadas, según test LSD al $5 \%$. 
Tabla 3. Matriz de correlación de Spermann $(\mathrm{n}=12)$ entre diversidad $\left(\mathrm{J}_{\mathrm{d}}\right)$, contribución de Carex $\operatorname{spp}\left(\right.$ Carex $\left.\mathrm{spp}_{\mathrm{d}}\right)$ y contribución de Eleocharis pseudoalbibracteata (E. pseudo $\mathrm{d}_{\mathrm{d}}$ ) de la dieta del piuquén (Chloephaga melanoptera Eyton, 1838) y diversidad $\left(\mathrm{J}_{\mathrm{veg}}\right)$, producción de materia seca (PMS, $\left.\mathrm{kg} \mathrm{MS} \mathrm{ha}^{-1}\right)$, contribución de Carex spp (Carex $\mathrm{spp}_{\mathrm{veg}}$ ) y contribución de Eleocharis pseudoalbibracteata $\left(\right.$ E. pseudo ${ }_{\mathrm{veg}}$ ) del pastizal. Cuncumén, IV Región de Coquimbo, comuna de Salamanca, Chile.

\begin{tabular}{|c|c|c|c|c|c|}
\hline & \multicolumn{4}{|c|}{ Pastizal } \\
\hline & & $\mathbf{J}_{\mathrm{veg}}$ & PMS & Carex $\mathrm{spp}^{1} \cdot \mathrm{veg}$ & E. pseudo. $_{\mathrm{veg}}$ \\
\hline \multirow{3}{*}{ Dieta } & $\mathrm{J}_{\mathrm{d}}$ & $-0,59^{*}$ & $-0,23^{\mathrm{ns}}$ & $0,09^{\mathrm{ns}}$ & $-0,48^{\mathrm{ns}}$ \\
\hline & Carex $\mathrm{spp}^{1} \cdot \mathrm{d}$ & $-0,71^{*}$ & $0,22^{\mathrm{ns}}$ & $-0,22^{\mathrm{ns}}$ & $-0,67^{*}$ \\
\hline & E. pseudo. $\cdot_{\mathrm{d}}$ & $0,80^{*}$ & $-0,11^{\mathrm{ns}}$ & $-0,09^{\mathrm{ns}}$ & $0,75^{*}$ \\
\hline
\end{tabular}

1 Carex spp. se refiere a la suma de las especies Carex gayana Desv., Carex maritima Gunn. y Carex vallis-pulchrae Phil.

* Correlación significativa considerando $\mathrm{p} \leq 0,05$.

ns Correlación no significativa $\mathrm{p}>0,05$.

Tabla 4. Índice de selectividad de Savage de las principales especies vegetales de la dieta del piuquén (Chloephaga melanoptera Eyton, 1838) en Cuncumén, IV Región de Coquimbo, comuna de Salamanca, Chile (Temporadas 2011-2012).

\begin{tabular}{|c|c|c|c|c|}
\hline \multirow{2}{*}{ Especie vegetal } & \multicolumn{2}{|c|}{2011} & \multicolumn{2}{|c|}{2012} \\
\hline & $\mathrm{W}_{\mathrm{i}}$ & Selección $^{1}$ & $\mathrm{~W}_{\mathrm{i}}$ & Selección ${ }^{1}$ \\
\hline Lobelia oligophylla Wedd. & 0,027 & rechaza & 0,047 & rechaza \\
\hline Festuca werdermannii St.-Yves. & 0,109 & rechaza & 0,000 & rechaza \\
\hline Eleocharis pseudoalbibracteata Nees \& Meyen ex Kunth. & 0,136 & rechaza & 1,421 & selecciona 1 \\
\hline Deyeuxia chrysostachya E. Desv. & 0,163 & rechaza & 0,053 & rechaza \\
\hline Hordeum pubiflorum Hook F. & 0,335 & rechaza & 10,959 & selecciona ${ }^{1}$ \\
\hline Cerastium humifusum Cambess. ex A. St.-Hil. & 0,621 & rechaza & 1,455 & selecciona \\
\hline Carex $\mathrm{spp}^{2}$. & 0,952 & rechaza & 0,673 & rechaza $^{1}$ \\
\hline Trifolium repens $\mathrm{L}$. & 0,977 & rechaza & 0,051 & rechaza \\
\hline Patosia clandestina (Phil.) Buchenau. & 1,222 & selecciona & 0,793 & rechaza \\
\hline Mimulus depressus Phill. & 1,390 & selecciona & 0,000 & rechaza \\
\hline Werneria pygmaea Gillies ex Hook. \& Arn. & 1,495 & selecciona & 0,000 & rechaza $^{1}$ \\
\hline Deschampsia caespitosa (L.) P. Beauv. & 2,320 & selecciona & 0,026 & rechaza \\
\hline Juncus arcticus var. Andicola Willd. & 6,328 & selecciona $^{1}$ & 0,015 & rechaza $^{1}$ \\
\hline Zameioscirpus gaimarioides (É. Desv.) Dhooge \& Goetgh & 15,456 & selecciona $^{1}$ & 0,022 & rechaza $^{1}$ \\
\hline
\end{tabular}

1 En negrita resultados significativos $\left(\lambda^{2}=3,8415, \mathrm{p}=0,05\right)$.

2 Carex spp. Se refiere a la sumatoria de las especies Carex gayana Desv., Carex maritima Gunn. y Carex vallis-pulchrae Phil.

la tendencia lineal y significativa $(\mathrm{p}<0,0001)$ observada entre la contribución de esta especie vegetal en el pastizal y en la dieta (Figura 1). El valor mayor a uno de la pendiente de la recta en esta relación confirma la selección que se observa en esa especie vegetal por parte del piuquén, destacando su rol nutricional y aportando de forma importante tanto a la mantención como a procesos productivos, además de contribuir a proporcionar volumen a la dieta del ave.

Otras especies vegetales también presentaron alta selectividad, en especial en el caso de la gramínea Hordeum pubiflorum y los graminoides Juncus articus y Zameioscirpus gaimarioides. Sin embargo, y a diferencia de lo que ocurre con E. pseudoalbibracteata, su aporte, tanto en los pastizales como en la dieta, fue bajo $(<5 \%)$. Estas especies podrían cumplir roles como mejoradoras de la calidad de la dieta, ya que a pesar de estar presentes en una baja proporción en el pastizal, los herbívoros son capaces de seleccionarlas (Castellaro et al., 2020).

El pastoreo conjunto con herbívoros simpátricos de mayor tamaño corporal y con sistema digestivo 


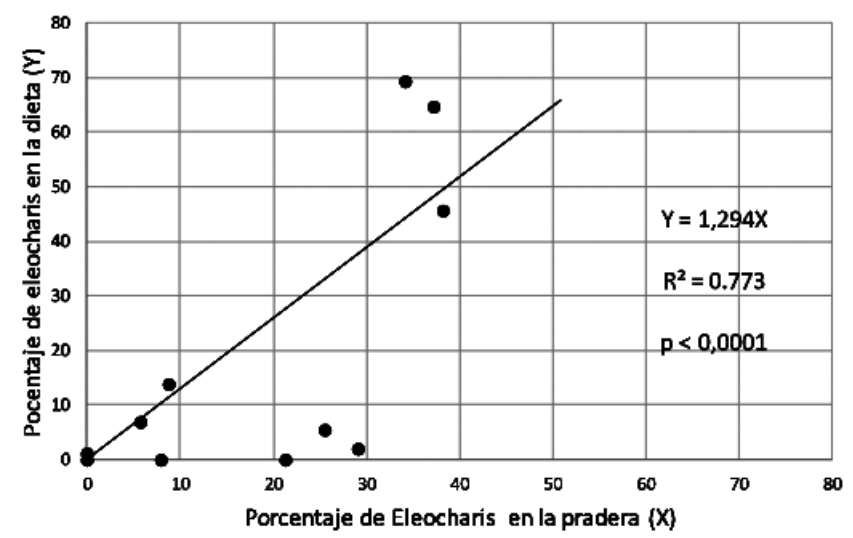

Figura 1. Regresión entre el porcentaje de Eleochasis albibracteata presente en la pradera y el porcentaje de la misma especie presente en la dieta del piuquén (Chloephaga melanoptera Eyton, 1838). La línea de regresión fue forzada a pasar por el origen. Línea pinteada señala la bisectriz.

más eficiente para la cosecha de especies vegetales más fibrosas (Edouard et al., 2010), podría beneficiar al piuquén dándole acceso a brotes tiernos, hierbas más bajas y con contenidos más altos de proteína (Ruifrok et al., 2015), aumentando la digestibilidad de su dieta y con ello la capacidad de uso de los nutrientes (Durant et al., 2004).

\section{Conclusiones}

En ambientes altoandinos el piuquén consume esencialmente especies graminoides, concentrando sus esfuerzos de forrajeo en especies vegetales con altos contenidos de proteína y bajos de fibra como Eleocharis pseudoalbibracteata. Esta especie cumpliría roles nutricionales de mantención y producción, así como un rol funcional, aportando volumen a la dieta del ave. Existe un grupo de especies que a pesar de estar presentes en baja proporción en los pastizales hidromórficos estudiados, son seleccionadas por el piuquén, contribuyendo probablemente a obtener una mejor calidad en su dieta.

\section{Agradecimientos}

Trabajo financiado por el Proyecto "CS-601 Estudio de Caracterización de los Humedales de la Cordillera Sur de Cuncumén. MINERA LOS PELAMBRES- AT-EME S.A.

\section{Literatura Citada}

Alisauskas R.T.; Ankneya, C.D.; Klaas, E.

1988. Winter diets and nutrition of midcontinental Lesser Snow Geese. J. Wildl. Manage, 52: 403-414.

Anderson, H.A.; Godfrey, T.G.; Woodin S.J.; van der Wal, R. 2012. Finding food in a highly seasonal landscape: where and how pink footed geese Anser brachyrhynchus forage during the Arctic spring. Journal of Avian Biology, 43: 415-422. A.O.A.C.

2000. Official methods of analysis of the AOAC International. $17^{\text {th }}$ ed. Association of Official Analytical Chemist. Ed. Arlington, USA.2200 p.

Barboza, P.; Parker, K.L.; Hume. D.

2009. Integrative Wildlife Nutrition. Ed. Springer. Berlín, Alemania. 342 p.

Castellaro, G.; Squella, F.; Muñoz, P.

2007. Efecto de la especie animal y densidad de carga sobre la utilización de un rastrojo de trigo por ovejas y alpacas durante el periodo seco-estival. III. composición botánica de la dieta e índices de selección de las principales especies consumidas. In: XXXII Reunión Anual de la Sociedad Chilena de Producción Animal A.G. (SOCHIPA), Frutillar, Chile. 14-16 de noviembre de 2007. SOCHIPA, Frutillar, Chile. pp. 23-24.

Castellaro, G.; Araya, R.

2012. Manejo de Praderas Altiplánicas: Antecedentes, Descripción, Evaluación y Manejo. Proyecto FIA (Fundación para la Innovación Agraria). Santiago de Chile, Chile. 65 p.

Castellaro, G.; Orellana, C.; Escanilla, J.; Bastías, C.; Cerpa, P.; Raggi, L.

2020. Botanical Composition and Diet Quality of the Vicuñas (Vicugna vicugna Mol.) in Highland Range of Parinacota, Chile. Animals, 10(7): 1205. doi.org/10.3390/ ani10071205 
Cochi, N.; Prieto, G.: Dangles, O.; Rojas, A.; Ayala, C.; Condori

B.; Casazola. J.L.

2014. Metodología para evaluar el potencial productivo y la dinámica. Ecología en Bolivia, 49: 120-131.

Doiron, M.; Gauthier, G.; Lévesque, E.

2014. Effects of experimental warming on nitrogen concentration and biomass of forage plants for an arctic herbivore. Journal of Ecology, 102: 508-517.

Durant, D.; Fritz H.; Duncan, P.

2004. Feeding patch selection by herbivorous Anatidae: the influence of body size, and of plant quantity and quality. Journal of Avian Biology, 35: 144-152.

García, J.E.; Torres, J.; García, F.T.

2015. Identificación de especies en los humedales altoandinos de la concesión para la Conservación Alto Huayabamba. Scientia, 7: 13-18.

Gastó J.; Cosio, F.; Panario, D.

1993. Clasificación de Ecorregiones y determinación de sitio y condición. Manual de Aplicación a municipios y predios rurales. Red de Pastizales Andinos. Santiago, Chile. 254 p.

Daget, P.H.; Poissonet, J.

1971. Une méthode d'analyse phytologique des prairies, critères d'application. Ann. Agron., 22: 5-41.

Garnick, S.; Barboza, P.S.; Walker, J.

2018. Assessment of Animal-Based Methods Used for Estimating and Monitoring Rangeland Herbivore Diet Composition. Rangeland Ecology \& Management, 71(2018): 449-457.

Gauthier, G.

1993. Feeding ecology of nesting Greater Snow Geese. J. Wildl. Manage, 57: 216-223.

Karmiris, I.; Platis, P.D.; Kazantzidis, S.; Papachristou, T.G. 2011. Diet Selection by Domestic and Wild Herbivore Species in a Coastal Mediterranean Wetland. Annales Zoologici Fennici, 48: 233-242.

López-Calleja, M.V.; Bozinovic, F.

2000. Energetics and nutritional ecology of small herbivorous birds. Revista Chilena de Historia Natural, 73: 411-420.

Le Houérou, H.N.

1984. Rain use efficiency a unifying concept in arid land ecology. J. Arid Environ., 7: 213-247.

Manly, B.F.J.; Mac Donald L.L.; Thomas, D.L.

1993. Resource selection by animals. Statistical design and analysis for field studies. Chapman \& Hall. London. UK. $222 \mathrm{p}$.

Ortega, I.; M. Berger; Flórez, M.

1993. Manual técnico de microhistología. Texas Tech University y Programa de Apoyo y Colaboración en la Investigación de Rumiantes Menores (SR-CRSP) en cooperación con el Instituto Boliviano de Tecnología Agropecuaria (IBTA). La Paz, Bolivia. 48 p.

Passera, C.B.; Dalmasso. A.D.; Borsetto, O.

1983. Método de Point Quadrat Modificado. Cap. 12, In: Taller de arbustos forrajeros para zonas áridas y semiáridas. $2^{\text {da }}$ Ed. Subcomité Asesor del Árido Subtropical Argentino. Amawald. S.A. Buenos Aires, Argentina. pp. 71-79.

Ruifrok, J.L.; Janzen, T.D.; Kuijper, J.P.; Rietkerk, M.; Olff, H.; Smit, C.

2015. Cyclical succession in grazed ecosystems: The importance of interactions between different-sized herbivores and different-sized predators. Theoretical Population Biology, 101: 31-39.

Savage, R.E.

1931. The relation between the feeding of the herring off the cast coast of England and the plankton the surrounding waters. Fishery Investigation, Ministry of Agriculture. Food and Fisheries. Series 2, 12: 1-88.

Smith, R.L.; Smith, T.M.

2012. Elements of Ecology, $8^{\text {th }}$ Edition. Pearson. Boston, USA. 704 p.

Squeo, F.A.; G. Barry; B.G. Warner; R. Aravena and D. Espinoza. 2006. Bofedales: high altitude peatlands of the central Andes. Revista Chilena de Historia Natural, 79: 245-255.

Summers, R.W. and G. Castro.

1988. Population size and feeding Cimbalar of Andean Geese at Lake Junin, Perú. Wildfowl, 39: 22-28.

Summers, R.W.; Grieve, A.

1982. Diet, Feeding Behaviour and Food Intake of the Upland Goose (Chloephaga picta) and Ruddy- Headed Goose ( $C$. rubidiceps) in the Falkland Islands. Journal of Applied Ecology, 19: 783-804.

Sedinger, J.S.

1997. Adaptations to and Consequences of an Herbivorous Diet in Grouse and Waterfowl. The Condor, 99: 314-326.

Tellería, J.L.; J.L. Venero and T. Santos.

2006. Conserving birdlife of Cimbalar highland bogs: effects of patch-size and habitat quality on species richness and bird numbers. Ardeola, 53: 271-283.

Wang X.; Y. Zhang; M. Zhao; L. Cao and A.D. Fox.

2013. The benefits of being big: effects of body size on energy budgets of three wintering goose species grazing Carex beds in the Yangtze River floodplain, China. J Ornithol, 154: 1095-1103. 
